\title{
Delineation of PIGV mutation spectrum and associated phenotypes in hyperphosphatasia with mental retardation syndrome
}

\author{
Denise Horn ${ }^{\star, 1}$, Dagmar Wieczorek ${ }^{2}$, Kay Metcalfe ${ }^{3}$, Ivo Barić ${ }^{4,5}$, Lidija Paležac ${ }^{5}$, Mario Cuk ${ }^{4,5}$, \\ Danijela Petković Ramadža ${ }^{4}$, Ulrike Krüger ${ }^{1}$, Stephanie Demuth ${ }^{6}$, Wolfram Heinritz ${ }^{7}$, Tobias Linden ${ }^{8}$, \\ Jens Koenig ${ }^{8}$, Peter N Robinson ${ }^{1}$ and Peter Krawitz ${ }^{1}$
}

Three different genes of the glycosylphosphatidylinositol anchor synthesis pathway, PIGV, PIGO, and PGAP2, have recently been implicated in hyperphosphatasia-mental retardation syndrome (HPMRS), also known as Mabry syndrome, a rare autosomal recessive form of intellectual disability. The aim of this study was to delineate the PIGV mutation spectrum as well as the associated phenotypic spectrum in a cohort of 16 individuals diagnosed with HPMRS on the basis of intellectual disability and elevated serum alkaline phosphate as minimal diagnostic criteria. All PIGV exons and intronic boundaries were sequenced in 16 individuals. Biallelic PIGV mutations were identified in 8 of 16 unrelated families with HPMRS. The most frequent mutation detected in about $80 \%$ of affected families including the cases reported here is the c.1022C $>$ A PIGV mutation, which was found in both the homozygous as well as the heterozygous state. Four further mutations found in this study (c. 176T $>$ G, c.53G $>$ A, c.905T $>$ C, and c. $1405 \mathrm{C}>\mathrm{T}$ ) are novel. Our findings in the largest reported cohort to date significantly extend the range of reported manifestations associated with PIGV mutations and demonstrate that the severe end of the clinical spectrum presents as a multiple congenital malformation syndrome with a high frequency of Hirschsprung disease, vesicoureteral, and renal anomalies as well as anorectal malformations. PIGV mutations are the major cause of HPMRS, which displays a broad clinical variability regarding associated malformations and growth patterns. Severe developmental delays, particular facial anomalies, brachytelephalangy, and hyperphosphatasia are consistently found in PIGV-positive individuals.

European Journal of Human Genetics (2014) 22, 762-767; doi:10.1038/ejhg.2013.241; published online 16 October 2013

Keywords: Hyperphosphatasia; intellectual disability; alkaline phosphatase; brachytelephalangy; seizures

\begin{abstract}
INTRODUCTION
Hyperphosphatasia-mental retardation syndrome (HPMRS, MIM 239300, MIM 214749, and MIM 614207), which is also referred to as Mabry syndrome, is a recently delineated autosomal recessive form of intellectual disability with facial dysmorphism, seizures, consistently elevated serum alkaline phosphatase (AP) (hyperphosphatasia), and brachytelephalangy. HPMRS was recently shown to be caused by homozygous and compound heterozygous mutations in PIGV (MIM 610274), PIGO (MIM 614730), and PGAP2 (MIM 615187), which are members of the glycosylphosphatidylinositol (GPI) anchor synthesis pathway. ${ }^{1-4}$ The transcripts altered by PIGV and PIGO mutations are either aberrantly spliced, decrease the membrane stability of the protein, or impair enzyme function This leads to a defect in GPI anchor synthesis with a corresponding reduction in the level of GPI-anchored substrates such as AP localized at the cell surface. ${ }^{3,4}$ The third gene, $P G A P 2$, is involved in fatty acid remodeling of the GPI anchor, which is required for stable association of GPI-anchored proteins with the cell surface membrane rafts. $^{2}$
\end{abstract}

PIGV mutations as one of the causes of HPMRS have been reported in eight affected families so far. ${ }^{3,5,6}$ In two families with HPMRS, PIGO mutations have been identified; in two further families PGAP2 mutations have been found. ${ }^{2,4}$ PGAP2 mutations have also been found to be associated with nonspecific autosomal recessive intellectual disabilities in two consanguineous families. ${ }^{1}$

To determine the variability of clinical manifestations and the spectrum of PIGV mutations associated with this condition, we investigated a cohort of patients with HPMRS, with a range of ethnic origins.

\section{MATERIALS AND METHODS}

Patients

In this study, we included 16 individuals with intellectual disability and elevated serum alkaline phosphate in the mutational analysis of PIGV PIGV-negative cases were subject to a screen of all genes of the GPI anchor biosynthesis pathway by targeted sequencing as described in $^{2}$ (data not shown). The cohort carrying PIGV mutations consisted of nine patients from eight families (five male and four female patients, ranging in age from newborn to 19 years). All of these families except one were non-consanguineous

${ }^{1}$ Institut für Medizinische Genetik und Humangenetik, Charité Universitätsmedizin Berlin, Berlin, Germany; ${ }^{2}$ Institut für Humangenetik Essen, Universitätsklinikum Essen, Essen, Germany; ${ }^{3}$ Clinical Genetics, Genetic Medicine, St Mary's Hospital, Manchester, UK; ${ }^{4}$ Department of Pediatrics, University Hospital Center Zagreb, Zagreb, Croatia; ${ }^{5}$ University of Zagreb, School of Medicine, Zagreb, Croatia; ${ }^{6}$ Praxis für Humangenetik Erfurt, Erfurt, Germany; ${ }^{7}$ Praxis für Humangenetik Cottbus, Cottbus, Germany; ${ }^{8}$ Klinik für Kinder- und Jugendmedizin, Universitätsklinikum Münster, Münster, Germany

*Correspondence: Dr D Horn, Institut für Medizinische Genetik und Humangenetik, Charité Universitätsmedizin Berlin, Berlin, Augustenburger Platz 1 13353, Germany. Tel: 4930 450569132; Fax: 4930 450569914; E-mail: denise.horn@charite.de

Received 5 June 2013; revised 13 September 2013; accepted 18 September 2013; published online 16 October 2013 


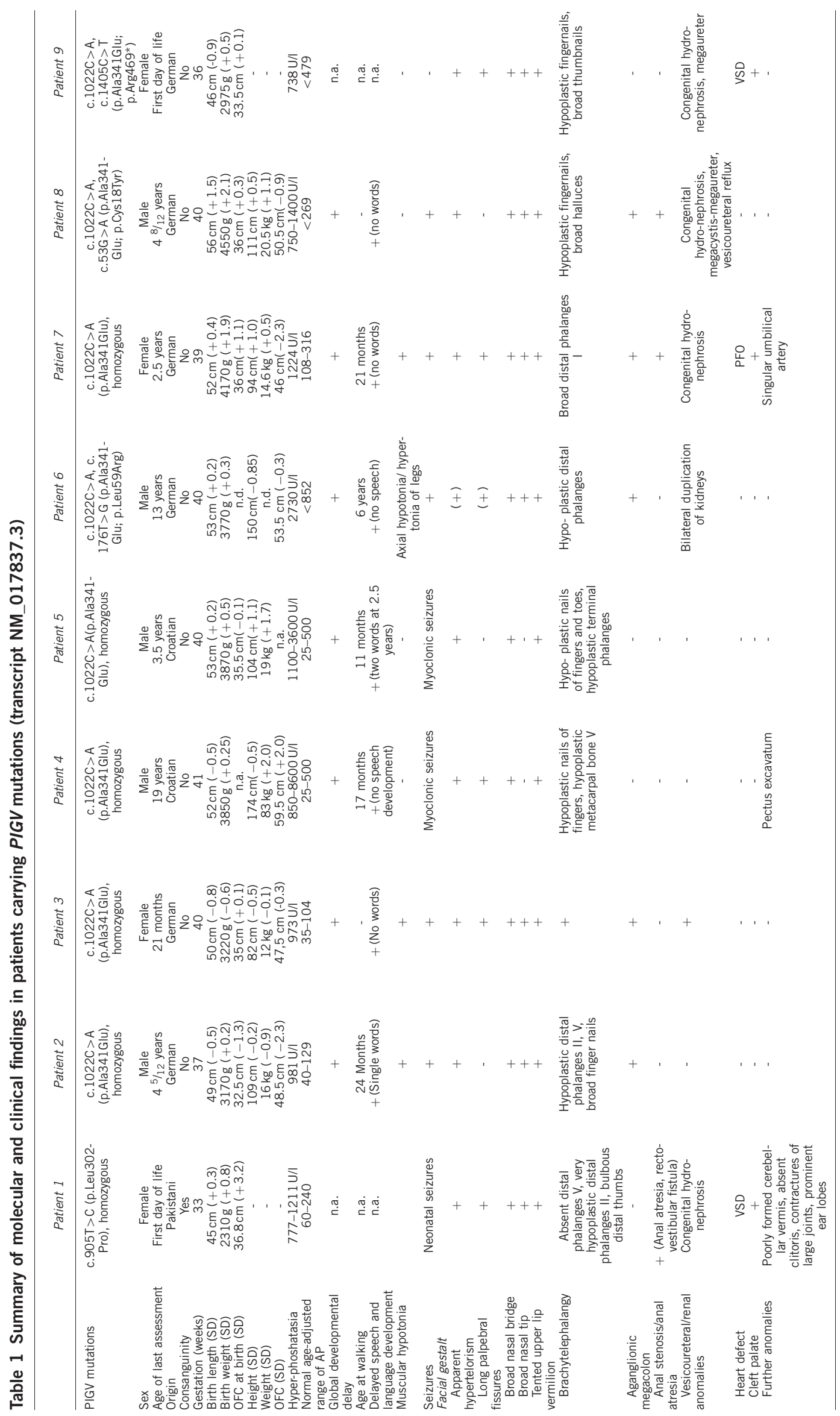


families from Germany and Croatia. One consanguineous family was from Pakistani descent and had one affected child. Clinical assessments were carried out on all patients by at least one of the authors. We obtained patients' DNA samples from the peripheral blood as well as parental DNA samples from all patients.

\section{Informed consent}

Written informed consent was given by the parents for genetic testing and publication of images. This study was approved by the Charite University Medicine ethics board.

\section{Mutational analysis}

Genomic DNA was isolated from the peripheral blood using standard techniques. For mutation screening, we amplified the coding region of PIGV (NM_017837.2), including the flanking intronic sequences and the predicted promoter region. Primer sequences and PCR conditions are available on request. PCR products were purified using the enzymes exonuclease I and shrimp AP treatment, and directly sequenced with the BigDye Terminator v3.1 Cycle Sequencing Kit (Applied Biosystems, Carlsbad, CA, USA) and analyzed on an automated DNA Analyzer (3730 Applied Biosystems). We submitted the variants detected in LOVD (http://www.lovd.nl) and GeneTalk (http:// www.gene-talk.de).

\section{RESULTS}

\section{Molecular data}

Mutational analysis of PIGV revealed homozygous or compound heterozygous mutations in the nine affected individuals from eight families; four of these mutations were novel (Table 1). The homozygous c.1022C $>$ A mutation located in exon 3 was repeatedly found in four families. Three patients (patients 6, 8, and 9) carried compound heterozygous mutations, with the c.1022C $>\mathrm{A}$ mutation as one mutation and c. $176 \mathrm{~T}>\mathrm{G}, \mathrm{c} .53 \mathrm{G}>\mathrm{A}$, and $\mathrm{c} .1405 \mathrm{C}>\mathrm{T}$ as the other. In patient 1 from Pakistani origin, we identified the homozygous c.905T $>\mathrm{C}$ mutation. All missense mutations affect evolutionarily highly conserved residues of PIGV (Figure1). Analysis of the samples of the parents showed heterozygous mutations in each case.

\section{Clinical data}

In our series of nine PIGV-positive patients with HPMRS from eight families, we observed marked variability regarding the presence of malformations (Table 1, Figures 2-4).

All patients had a global developmental delay. In all but two who started to walk normally, the age at which they first started to walk was delayed between 21 months and 6 years. Five individuals did not achieve verbal communication, and two individuals used single words only. All but one individuals developed seizures with variable onset and severity. Individual 1 developed tonic-clonic seizures shortly after birth and died due to sepsis/meningitis at the fourth day of life. Patient 9 was examined at her first day of life and did not present epilepsy at that time. Muscular hypotonia was found in four individuals.

The distinct pattern of facial anomalies present in all patients consisted of wide-set eyes, often with a large appearance, a short nose with a broad nasal bridge and tip, and a tented upper lip (Figures 2 and 4). Most showed up-slanting palpebral fissures.

At birth, normal growth parameters and head circumferences were observed in all except two (patients 7 and 8) who presented with overweight and one patient (patient 1) in whom macrocephaly was documented. During infancy and childhood, growth parameters were found to be in the normal range (height between -0.85 and +1.1 SD, weight between -0.9 and +2 SD) in all. Development of the postnatal occipitofrontal circumference (OFC) ranges between -2.3 and +2 SD with two affected individuals who showed microcephaly.

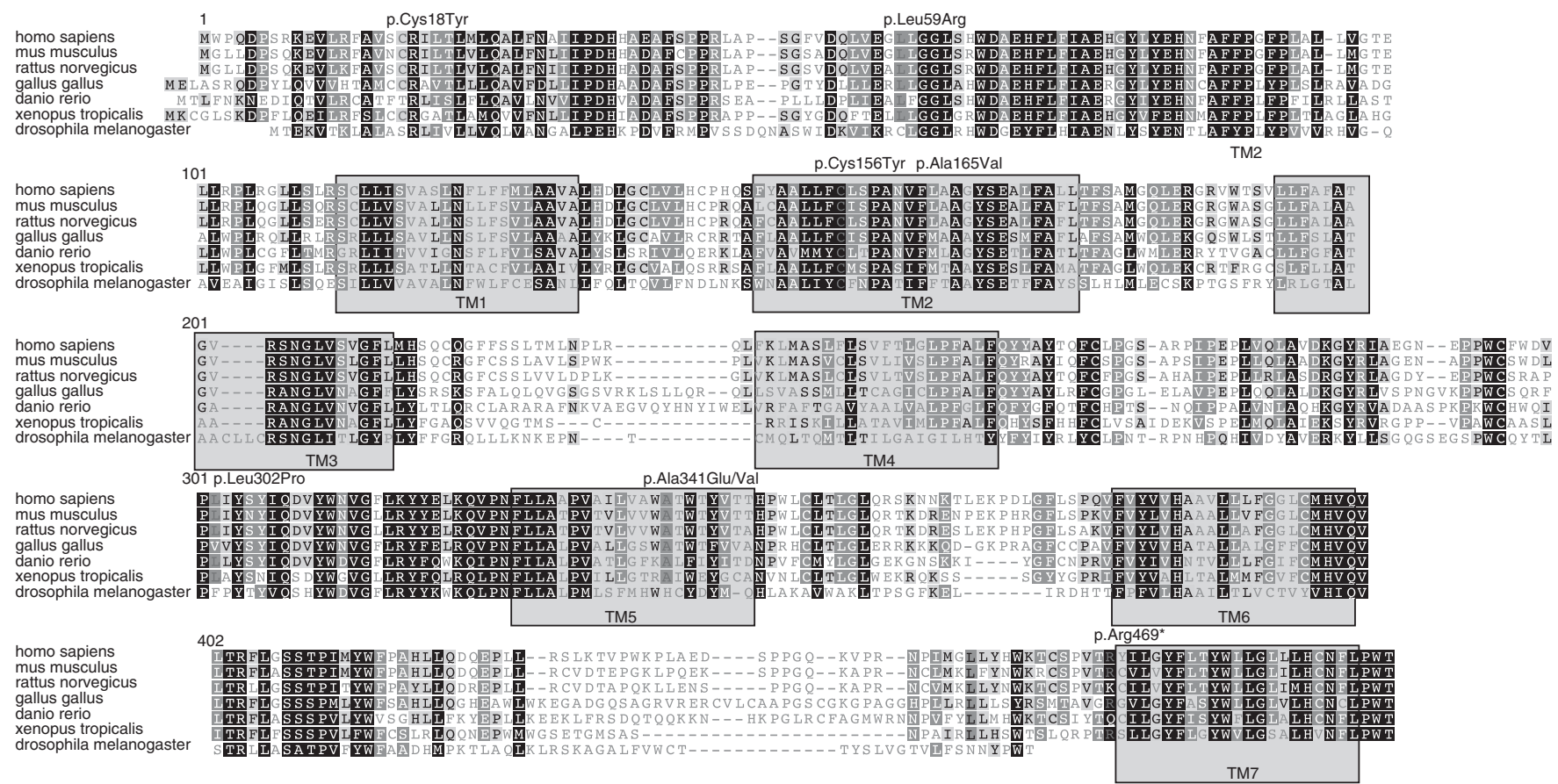

Figure 1 Multiple species alignment of PIGV with an overview of the positions of the detected mutations in the current cohort and in the literature. PIGV is a mannosyltransferase with seven predicted transmembrane domains (TM1-7) that is located in the ER. PIGV is involved in the early steps of GPI-anchor synthesis and is highly conserved in multicellular organisms. All depicted mutations affect protein stability and impair the transfer of the second mannose residue during GPI-anchor synthesis. In a homozygous or compound heterozygous state, these mutations result in reduced surface expression of GPIanchored proteins on the plasma membrane, which causes HPMRS. 


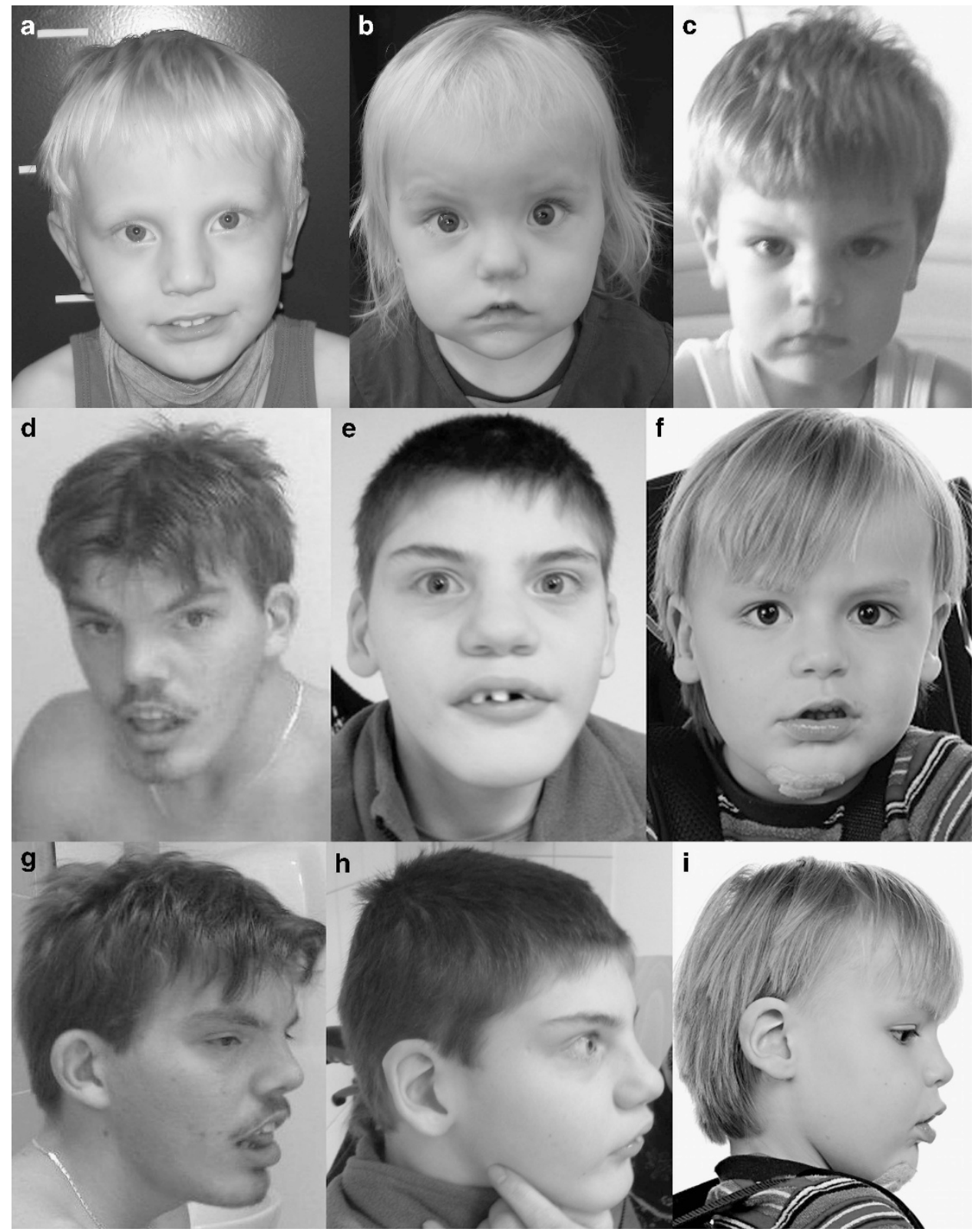

Figure 2 Facial photographs of individuals harboring PIGV mutations. Shown are patients 2 (a) at age of 4.5 years, 3 (b) at age of 21 months, 5 (c) at age of 3.5 years, $4(\mathbf{d}, \mathbf{g})$ at age of 19 years, $6(\mathbf{e}, \mathbf{h})$ at age of 13 years and $8(\mathbf{f}, \mathbf{i})$ at age of 4.5 years.

Elevated values of AP were a consistent finding. The degree of persistent hyperphosphatasia varied between about 1.5 and 17 times of the age-adjusted upper limit of the normal range in the individuals reported here.

All patients studied here had a variable degree of distal hand anomalies, namely brachytelephalangy (Figures 3 and 4). Eight of nine patients showed hypoplastic finger- and toenails as well as hypoplastic distal phalanges on the hand X-rays. One (patient 7) only displayed broad and short distal phalanges of the thumbs and great toes including short and broad corresponding nails of the affected digits. This clinical sign was also noted in patients 1,8 , and 9 in addition to brachytelephalangy of the other digits. Apart from brachytelephalangy patient 4 showed a hypoplastic metacarpal bone $\mathrm{V}$.
In five of the nine patients, Hirschsprung disease was diagnosed. Vesicoureteral and renal anomalies, for example, congenital hydronephrosis, were documented in six of the nine patients. Anorectal anomalies such as anal atresia or anorectal fistula were found in three of the nine affected individuals. In addition, it was observed that three patients had a cleft palate, whereas further three patients had a heart defect.

In patient 1 , MRI revealed a brain anomaly, namely a poorly formed cerebellar vermis. When the brain MRI scans of other patients reported here were available, normal findings excluded brain anomalies.

Patients 2 and 3 are siblings, whereas the others - except patient 6 - are the only affected individuals in their families. In the family of patient 6 , the first child was affected with Hirschsprung disease, 
vesicoureteral reflux, ectopia of the urethra, and an ileocolic mesentery commune. This male child died at the age of 8 months. In the second pregnancy, the female fetus presented with hydronephrosis, megaureter, and bilateral cleft lip-palate. The parents decided to terminate the pregnancy at the 21st week of gestation. The fetus of the third pregnancy was affected with a hypoplastic left heart and the parents again terminated the pregnancy. Samples of these affected fetuses were not available for PIGV analysis but because of the patterns of malformations, the fetuses of the first and second pregnancies have to be regarded as being probably affected with HPMRS.

\section{DISCUSSION}

In this study, different missense mutations as well as one stop mutation in PIGV were detected in about $50 \%$ of affected HPMRS families in our current cohort. Further mutations of PIGO and PGAP2 were not identified by targeted sequencing of all GPI anchor genes (data not shown). Therefore, the PIGV gene seems to be the most frequently mutated gene in this group of patients. Overall, six patients with homozygous mutations and three with compound heterozygous PIGV mutations were observed. Each mutation co-segregated in the respective family.

The known missense mutation c.1022C $>$ A leads to the amino-acid substitution p.Ala341Glu. ${ }^{3}$ Chinese hamster ovary $(\mathrm{CHO})$ cells transfected with the Ala341Glu mutant did not restore surface expression of GPI-anchored marker proteins indicating a reduced PIGV protein level. ${ }^{3}$ Therefore, this mutation predicts protein instability and represents a mutational hotspot as it is observed in a homozygous or compound heterozygous state in 13 of 16 of the molecularly proven familial cases including 7 families presented here. Of note, another missense mutation, c.1022C $>\mathrm{T}$, at the same position has been identified in three reported cases. ${ }^{3,5,6}$ It is striking that all the families of European descent except one carry at least one mutation at this position of the gene. The novel mutations, c. $176 \mathrm{~T}>\mathrm{G}, \mathrm{c} .53 \mathrm{G}>\mathrm{A}, \mathrm{c} .905 \mathrm{~T}>\mathrm{C}$, and c.1405C $>$ T, were not found in the HapMap or the NCBI dbSNP database. The three novel missense mutations also predict substitutions of amino acids, which are evolutionarily conserved (Figure1). So far, with one exception (patient 9) only missense mutations of PIGV have been identified.

It has been hypothesized that a complete deficiency in GPI-anchor biosynthesis is not compatible with embryonic development. ${ }^{7}$ Up to date, all mutations of HPMRS patients that have been analyzed in $\mathrm{CHO}$ cells had residual protein function. Even in the severely affected patient (patient 9) with the heterozygous nonsense mutation, we do not suspect a complete loss of GPI-anchor synthesis: The preterminal stop codon is in the last exon of PIGV and will therefore not cause a nonsense-mediated decay of the transcript, and the second PIGV allele with the c.1022C $>\mathrm{T}$ missense mutation results in a protein with residual function. ${ }^{3}$

HPMRS could present as apparently non-syndromic form of intellectual disability at one end of the clinical spectrum but also as a multiple congenital malformation syndrome at the other end of this broad clinical spectrum. Six of nine affected individuals studied here showed two or more congenital malformations, whereas only two had no congenital anomalies. The most frequent anomalies are Hirschsprung disease as well as vesicoureteral and renal anomalies.

The degree of persisting hyperphosphatasia varies considerably in our study group.

Normal and even high growth parameters are observed in all patients at birth. No specific growth patterns including development

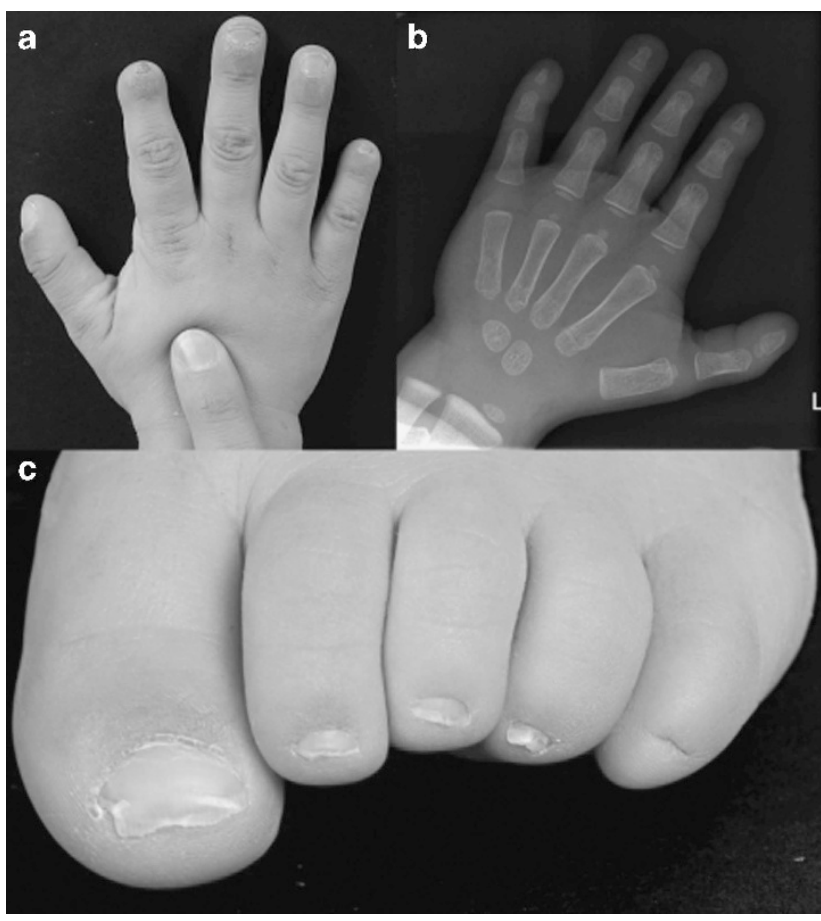

Figure 3 Hand anomalies of patients with PIGV mutations. (a) Brachytelephalangy with missing and hypoplastic nails of fingers of patient 8. (b) Hand radiograph of patient 2 showing hypoplastic distal phalanges. (c) Broad hallux and hypoplastic toenails of patient 8 .
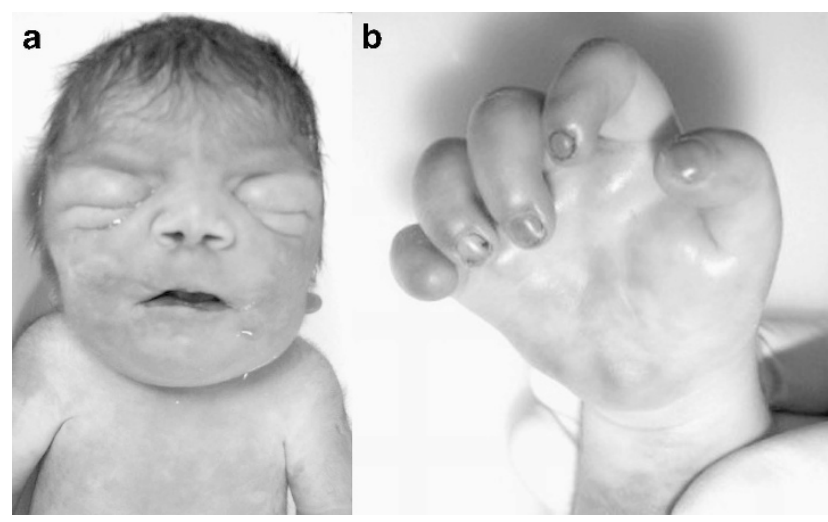

Figure 4 Affected newborn (patient 1) that died shortly after birth (a) Facial photograph showing broad nasal bridge, a short nose, and a tented upper lip. (b) Hypoplastic fingernail of the index finger and aplasia of fingernail of the fifth digit.

of OFC are recognizable in our series; however, mild microcephaly as well as macrocephaly may occur.

Marked developmental delays with particularly delayed speech and language development were observed in all individuals studied here. Most of them did not develop speech and language skills, whereas two patients showed very limited expressive language development.

Seizures, which could even start in the newborn period, occurred in all but one of the individuals studied here, but are not present in all mutational proven cases. ${ }^{6}$ Muscular hypotonia is documented in half of our study cohort.

Despite some variability in the facial appearance, craniofacial abnormalities including wide-set eyes, a short nose with a broad 
nasal bridge and tip, and a tented upper lip were seen in all patients (Figures 2 and 3). However, facial dysmorphism may be subtle.

Brachytelephalangy varies in degree of shortness: the finger- and toenails could be hypoplastic or even absent in most of the patients; in a few affected individuals only the nails, in particular the thumbnail, is broad and short. Brachytelephalangy has to be regarded as one of the major clinical signs. However, it could be a subtle finding in some affected individuals. Only one reported patient (patient $4 \mathrm{in}^{5}$ ) did not present with brachytelephalangy. Of note, in this patient only one missense variant in PIGV was found, which did not show a functional effect (data not shown). ${ }^{5}$

In conclusion, severe developmental delay, the particular facial gestalt, and hyperphosphatasia constitute this distinct syndrome. A variable degree of brachytelephalangy has been observed in all molecularly confirmed cases. Seizures occur in a very high frequency. Hirschsprung disease, vesicoureteral, and renal anomalies as well as anorectal malformations should be considered as part of the clinical spectrum. Cleft palate and heart defects may be associated with PIGV mutations.

In this work, we present the most comprehensive collection of pathogenic mutations in PIGV and a detailed clinical description of the patients. We hope that a functional workup of these mutations with respect to their protein impairment of PIGV will enable a better prediction of the phenotypic outcome of the patients based on the genotype information.

\section{CONFLICT OF INTEREST}

The authors declare no conflict of interest.

\section{ACKNOWLEDGEMENTS}

We thank the patients and their families for participating. We acknowledge $\mathrm{P}$ Meinecke for critical review of the manuscript. This work was by supported by grants from the Ministry of Science, Education and Sport, Republic of Croatia, grant No: 108-1081870-1885 to IB, LP, MĆ, and DPR.

1 Hansen L, Tawamie H, Murakami Y et al: Hypomorphic mutations in PGAP2, encoding a GPI-anchor-remodeling protein, cause autosomal-recessive intellectual disability. Am J Hum Genet 2013; 92: 575-583.

2 Krawitz PM, Murakami Y, Riess A et al: PGAP2 mutations, affecting the GPI-anchorsynthesis pathway, cause hyperphosphatasia with mental retardation syndrome. Am J Hum Genet 2013; 92: 584-589.

3 Krawitz PM, Schweiger MR, Rödelsperger $C$ et al: Identity-by-descent filtering of exome sequence data identifies PIGV mutations in hyperphosphatasia mental retardation syndrome. Nat Genet 2010; 42: 827-829.

4 Krawitz PM, Murakami Y, Hecht J et al: Mutations in PIGO, a member of the GPI-anchor-synthesis pathway, cause hyperphosphatasia with mental retardation. Am J Hum Genet 2012: 91: 146-151.

5 Thompson MD, Roscioli T, Marcelis C et al: Phenotypic variability in hyperphosphatasia with seizures and neurologic deficit (Mabry syndrome). Am J Med Genet A 2012; 158A: 553-558.

6 Horn D, Krawitz P, Mannhardt A, Korenke GC, Meinecke P: Hyperphosphatasia-mental retardation syndrome due to PIGV mutations: expanded clinical spectrum. Am J Med Genet 2011; 155A: 1917-1922.

7 Nozaki M, Ohishi K, Yamada N, Kinoshita T, Nagy A, Takeda J: Developmental abnormalities of glycosylphosphatidylinositol-anchor-deficient embryos revealed by Cre/loxP system. Lab lvest 1999; 79: 293-299. 